\title{
Sea quark QED effects and twisted mass fermions
}

\section{R. Frezzotti*}

Dipartimento di Fisica, Università di Roma "Tor Vergata" and INFN, Sezione di Roma 2

E-mail: frezzottieroma2.infn.it

\section{G.C. Rossi}

Dipartimento di Fisica, Università di Roma "Tor Vergata" and INFN, Sezione di Roma 2 Centro Fermi - Museo Storico della Fisica, Piazza del Viminale 1 - 00184 Roma, Italy

E-mail: rossig@roma2.infn.it

\section{N. Tantalo}

Dipartimento di Fisica, Università di Roma "Tor Vergata" and INFN, Sezione di Roma 2

E-mail: nazario.tantalo@roma2.infn.it

We show that maximally twisted mass fermions can be employed to regularize on the lattice the fully unquenched QCD+QED theory with vanishing $\theta$-term. We discuss how the critical mass of the up and down quarks can be conveniently determined beyond the electroquenched approximation by imposing that certain symmetries of continuum QCD+QED, which are broken by Wilson terms, get restored in the continuum limit. A mixed action setup is outlined that allows to extend beyond the electroquenched approximation the computation (with only $\mathrm{O}\left(a^{2}\right)$ artifacts) of the leading isospin breaking corrections to physical observables using the RM123 method and (pure QCD) ETMC gauge ensembles with $N_{f}=2+1+1$ dynamical quark flavours.

34th annual International Symposium on Lattice Field Theory

24-30 July 2016

University of Southampton, UK

\footnotetext{
* Speaker.
} 


\section{Introduction}

In refs. [1, 2] a strategy for evaluating the leading isospin breaking (LIB) corrections to hadronic quantities has been proposed. It is based on expanding the full $\mathrm{Q}(\mathrm{C}+\mathrm{E}) \mathrm{D}$ observables to first order in powers of the small quantities $\left(m_{d}-m_{u}\right) / \Lambda_{Q C D}$ and $\alpha_{e m}$ (so-called RM123 approach). In this way the computational task is reduced to that of evaluating hadronic correlators with insertions of electromagnetic (e.m.) currents or quark scalar densities in the theory with no electromagnetism and no $u-d$ mass splitting (isosymmetric theory) .

In the same papers the viability of the RM123 approach was tested by evaluating the LIB corrections to hadron masses as well as the Dashen's theorem breaking parameter $\varepsilon_{\gamma}$ using the $N_{f}=2$ ensembles generated by ETMC [3,4]. The study of LIB effects in the leptonic meson decay rates, which was started in ref. [2] as far as the correction $\propto\left(m_{d}-m_{u}\right)$ is concerned, has been recently extended to the LIB e.m. corrections (see talks by Tantalo [5] and Simula [6] for the method and preliminary results) by following the general strategy of ref. [7], which allows to keep under control the infrared divergencies arising in the intermediate steps of the calculation.

All these investigations have been carried out so far in the electro-quenched approximation, i.e. by treating sea quarks as if they were electrically neutral (diagrammatically this means neglecting all contributions with photons attached to sea quark loops). Though this is a reasonable first approximation, it appears difficult to reliably control the systematic error it induces on the computed LIB effects. Here we discuss how twisted mass LQCD $[8,9,10]$ can be conveniently combined with the RM123 approach in order to evaluate ${ }^{1}$ LIB corrections to hadronic observables beyond the electro-quenched approximation in the theory with dynamical $u, d$ as well as $s$ and $c$ quarks.

\section{A lattice regularization of $\mathrm{Q}(\mathrm{C}+\mathrm{E}) \mathrm{D}$ with maximally twisted Wilson fermions}

The use of maximally twisted Wilson fermions allows to avoid $\mathrm{O}(a)$ lattice artifacts in physical observables at the price of introducing parity and isospin breakings at finite lattice spacing, which come on top of the physical isospin violations. One may thus worry that in the presence of e.m. interactions a delicate tuning of bare mass parameters is needed in order to obtain a continuum effective action with no strong and e.m. $\theta$-terms. We show here that this is not the case if one works at maximal twist, i.e. with $M_{0}=M_{c r}$ for each quark pair.

We discuss the conceptual point in $\mathrm{LQ}(\mathrm{C}+\mathrm{E}) \mathrm{D}$ with $u$ and $d$ (non-degenerate) quarks. The quark lattice action of $\mathrm{Q}(\mathrm{C}+\mathrm{E}) \mathrm{D}$ for a maximally twisted isospin doublet $\psi=\left(\psi_{u}, \psi_{d}\right)$ reads [11]

$$
\begin{gathered}
S_{\mathrm{F}}^{\mathrm{Q}(\mathrm{C}+\mathrm{E}) \mathrm{D}}(\psi, \bar{\psi}, U, E)=a^{4} \sum_{x} \bar{\psi}(x)\left[\gamma \cdot \widetilde{\nabla}-i \gamma_{5} \tau_{3} W_{\mathrm{cr}}+\mu+\tau_{3} \varepsilon\right] \psi(x) \\
\gamma \cdot \widetilde{\nabla} \equiv \frac{1}{2} \sum_{\mu} \gamma_{\mu}\left(\nabla_{\mu}^{\star}+\nabla_{\mu}\right), \quad W_{\mathrm{cr}} \equiv-\frac{a}{2} \sum_{\mu} \nabla_{\mu}^{\star} \nabla_{\mu}+M_{\mathrm{cr}} \\
\nabla_{\mu} \psi(x) \equiv \frac{1}{a}\left[\mathscr{U}_{\mu}(x) \psi(x+a \hat{\mu})-\psi(x)\right], \quad \nabla_{\mu}^{*} \psi(x) \equiv \frac{1}{a}\left[\psi(x)-\mathscr{U}_{\mu}^{\dagger}(x-a \hat{\mu}) \psi(x-a \hat{\mu})\right] \\
\mathscr{U}_{\mu}(x)=E_{\mu}(x) U_{\mu}(x), \quad E_{\mu}(x)=\frac{11+\tau_{3}}{2} e^{i e q_{u} \triangleleft_{\mu}(x)}+\frac{11-\tau_{3}}{2} e^{i e q_{d} \mathscr{\vartheta}_{\mu}(x)},
\end{gathered}
$$

\footnotetext{
${ }^{1}$ From the numerical viewpoint it is assumed (with no discussion here) that suitable methods are employed to evaluate the fermionically disconnected diagrams that arise when accounting for electro-unquenched LIB effects.
} 
where $U_{\mu}(x)$ and $E_{\mu}(x)$ are the strong and e.m. (non-compact QED) gauge links. The flavour structure of the latter is due to the unequal electric charges of up and down quarks. In flavour space the charge operator reads $Q=e\left(11 / 6+\tau_{3} / 2\right)$, where $e$ is the electric charge. Consequently also the critical mass counterterm, $M_{c r}$, will take the diagonal matrix form

$$
M_{c r}=M_{c r}^{u} \frac{1+\tau_{3}}{2}+M_{c r}^{d} \frac{11-\tau_{3}}{2} \equiv m_{c r} 1+\tilde{m}_{c r} \tau_{3} .
$$

We stress that in eq. (2.1) the critical Wilson term, $-\bar{\psi} i \gamma_{5} \tau^{3} W_{c r} \psi$, is chirally twisted in the $\tau_{3}$ "isospin direction" to comply with e.m. gauge invariance, which leads to a complex quark determinant (see sect. 4 on how the resulting problem can be circumvented in the RM123 approach).

For convenience we separate out quark flavours and rewrite eq. (2.1) in the case of generic twist angles, called $\theta_{u}$ and $\theta_{d}$ (maximal twist is recovered e.g. for $\theta_{u}=-\theta_{d}=\pi / 2$ ), obtaining

$$
\begin{aligned}
& S^{L Q(C+E) D}(\psi, \bar{\psi}, U, E)=a^{4} \sum_{x}\left[\left.F F\right|_{E}(x)+\left.\operatorname{tr}(G G)\right|_{U}(x)\right]+ \\
& +a^{4} \sum_{x} \bar{\psi}_{u}(x)\left[\gamma \cdot \widetilde{\nabla}^{u}+e^{-i \theta_{u} \gamma_{5}} W_{\mathrm{cr}}^{u}+M_{u}\right] \psi_{u}(x)+a^{4} \sum_{x} \bar{\psi}_{d}(x)\left[\gamma \cdot \widetilde{\nabla}^{d}+e^{-i \theta_{d} \gamma_{5}} W_{\mathrm{cr}}^{d}+M_{d}\right] \psi_{d}(x),
\end{aligned}
$$

where $M_{u / d}=\mu \pm \varepsilon$ are bare quark masses, $W_{\mathrm{cr}}^{f}=-\frac{a}{2} \sum_{\mu} \nabla^{* f} \nabla^{f}+M_{c r}^{f}$ and a superscript ${ }^{u}$ or $^{d}$ is attached to the covariant derivatives because $q_{u} \neq q_{d}$. Eq. (2.4) shows that $S^{L Q(C+E) D}$ is a periodic function of $\theta_{u}$ and $\theta_{d}$. Since lattice singlet axial rotations are not anomalous, twist phases can be freely moved from the Wilson to the mass terms by means of the axial rotations

$$
\begin{array}{lll}
\mathrm{U}_{A}^{u}(1): & \psi_{u}=e^{i \gamma_{5} \theta_{u} / 2} \chi_{u} & \bar{\psi}_{u}=\bar{\chi}_{u} e^{i \gamma_{5} \theta_{u} / 2} \\
\mathrm{U}_{A}^{d}(1): & \psi_{d}=e^{i \gamma_{5} \theta_{d} / 2} \chi_{d} & \bar{\psi}_{d}=\bar{\chi}_{d} e^{i \gamma_{5} \theta_{d} / 2}
\end{array}
$$

by which the lattice action (2.4) can be brought into the form (with untwisted Wilson terms $W_{\mathrm{cr}}^{u, d}$ )

$$
\begin{gathered}
S^{L Q(C+E) D}(\chi, \bar{\chi}, U, E)=a^{4} \sum_{x}\left[\left.F F\right|_{E}+\left.\operatorname{tr}(G G)\right|_{U}+\sum_{f=u, d}\left(\bar{\chi}_{f} \gamma \cdot \widetilde{\nabla}^{f} \chi_{f}+\bar{\chi}_{f} W_{\mathrm{cr}}^{f} \chi_{f}\right)\right](x)+\mathrm{MTs} \\
\operatorname{MTs}=a^{4} \sum_{x} \sum_{f=u, d} \bar{\chi}_{f}(x) e^{i \theta_{f} \gamma_{5}} M_{f} \chi_{f}(x) \equiv a^{4} \sum_{x}\left[m_{u} S_{\chi}^{u}(x)+i \mu_{u} P_{\chi}^{u}+m_{d} S_{\chi}^{d}(x)+i \mu_{d} P_{\chi}^{d}\right],
\end{gathered}
$$

where we have introduced the lattice bare quark mass parameters and density operators

$$
m_{f} \equiv M_{f} \cos \theta_{f}, \quad \mu_{f} \equiv M_{f} \sin \theta_{f} \quad \text { and } \quad S_{\chi}^{f} \equiv \bar{\chi}^{f} \chi^{f}, \quad P_{\chi}^{f} \equiv \bar{\chi}^{f} \gamma_{5} \chi^{f}, \quad f=u, d .
$$

Symmetries of the lattice action (2.7) and power counting arguments imply [12] that the local effective action of the corresponding continuum theory can be written in the form

$$
\begin{aligned}
& S_{\text {cont }}^{Q(C+E) D}\left(\chi^{c}, \bar{\chi}^{c}, A, \mathscr{A}\right)=\int d x\left[\left.F F\right|_{\mathscr{A}}(x)+\left.\operatorname{tr}(G G)\right|_{A}(x)+\left.\sum_{f=u, d} \bar{\chi}_{f} \gamma \cdot D^{f}\right|_{A, \mathscr{A}} \chi^{f}\right]+\mathrm{MTs}, \\
& \mathrm{MTs}=\int d x\left[\hat{m}_{u} \hat{S}_{\chi}^{u}+i \hat{\mu}_{u} \hat{P}_{\chi}^{u}+\hat{m}_{d} \hat{S}_{\chi}^{d}+i \hat{\mu}_{d} \hat{P}_{\chi}^{d}\right]
\end{aligned}
$$

where $\chi_{f}, \bar{\chi}_{f}, \mathscr{A}$ (photon) and $A$ (gluon) stand now for suitably normalized continuum fields and

$$
\hat{m}_{f}=m_{f}^{\mathrm{sub}}\left(Z_{S}^{f}\right)^{-1}, \quad \hat{\mu}_{f}=\mu_{f}^{\mathrm{sub}}\left(Z_{P}^{f}\right)^{-1} \quad \text { and } \quad \hat{S}_{\chi}^{f}=Z_{S}^{f} S_{\chi}^{f \text {,sub }}, \quad \hat{P}_{\chi}^{f}=Z_{P}^{f} P_{\chi}^{f, \text { sub }}, \quad f=u, d
$$

denote the renormalized mass parameters and quark densities. Due to differencies in the renormalization constants of the relevant flavour singlet and non-singlet densities which start at 2-loop level 
and imply $S_{\chi}^{u(d), \text { sub }}=S_{\chi}^{u(d)}\left(1+\rho_{u(d)}^{S}\right)+S_{\chi}^{d(u)} \tilde{\rho}_{d(u)}^{S}$ as well as $P_{\chi}^{u(d), \text { sub }}=P_{\chi}^{u(d)}\left(1+\rho_{u(d)}^{P}\right)+P_{\chi}^{d(u)} \tilde{\rho}_{d(u)}^{P}$, the multiplicatively renormalizable mass parameters take (see e.g. ref. [11]) the form

$$
m_{u(d)}^{\mathrm{sub}}=m_{u(d)}\left(1+\rho_{u(d)}^{m}\right)+m_{d(u)} \tilde{\rho}_{d(u)}^{m}, \quad \mu_{u(d)}^{\mathrm{sub}}=\mu_{u(d)}\left(1+\rho_{u(d)}^{\mu}\right)+\mu_{d(u)} \tilde{\rho}_{d(u)}^{\mu} .
$$

Noting that the twisted mass terms $i\left(\hat{\mu}_{u} \hat{P}_{\chi}^{u}+\hat{\mu}_{d} \hat{P}_{\chi}^{d}\right)$ and the action terms $\operatorname{tr}(\tilde{G} G)$ and $\tilde{F} F$ (which can only appear with coefficients $\propto \hat{\theta}_{f}$ being odd functions of $\hat{\mu}_{f} / \hat{m}_{f}, f=u, d$ ) are not independent operators in the continuum theory, as they are related by anomalous axial U(1) rotations of the quark fields (see below), one cheks that the form (2.10) of the continuum action is indeed the most general one. Trading $\hat{m}_{f}$ and $\hat{\mu}_{f}$ for the alternative renormalized parameters $\hat{M}_{f}$ and $\hat{\theta}_{f}(f=u, d)$,

$$
\hat{M}_{u}=\sqrt{\hat{m}_{u}^{2}+\hat{\mu}_{u}^{2}}, \quad \hat{M}_{d}=\sqrt{\hat{m}_{d}^{2}+\hat{\mu}_{d}^{2}}, \quad \tan \hat{\theta}_{u}=\frac{Z_{S}^{u}}{Z_{P}^{u}} \tan \theta_{u}, \quad \tan \hat{\theta}_{d}=\frac{Z_{S}^{d}}{Z_{P}^{d}} \tan \theta_{d},
$$

the continuum theory effective action (2.10) is rewritten as

$$
\begin{aligned}
& S_{\text {cont }}^{Q(C+E) D}\left(\chi^{c}, \bar{\chi}^{c}, A, \mathscr{A}\right)=\int d x\left[\left.F F\right|_{\mathscr{A}}(x)+\left.\operatorname{tr}(G G)\right|_{A}(x)\right]+ \\
& +\int d x \bar{\chi}_{u}(x)\left[\gamma \cdot D^{u}+e^{i \hat{\theta}_{u} \gamma_{5}} \hat{M}_{u}\right] \chi_{u}(x)+\int d x \bar{\chi}_{d}(x)\left[\gamma \cdot D^{d}+e^{i \hat{\theta}_{d} \gamma_{5}} \hat{M}_{d}\right] \chi_{d}(x) .
\end{aligned}
$$

In the formal continuum theory the analogs of the U(1)-axial rotations (2.5) and (2.6) are anomalous, so the effective action (2.15) can be equivalently cast into the form

$$
\begin{aligned}
& S_{\text {cont }}^{Q(C+E) D}(\psi, \bar{\psi}, A, \mathscr{A})=\int d x\left[\left.F F\right|_{\mathscr{A}}(x)+\left.\operatorname{tr}(G G)\right|_{A}(x)\right]+ \\
& +\int d x \bar{\psi}_{u}(x)\left[\gamma \cdot D^{u}+\hat{M}_{u}\right] \psi_{u}(x)+\int d x \bar{\psi}_{d}(x)\left[\gamma \cdot D^{d}+\hat{M}_{d}\right] \psi_{d}(x)+ \\
& +\frac{i}{32 \pi^{2}}\left(\hat{\theta}_{u}+\hat{\theta}_{d}\right) \int d x\left[\left.e^{2} F \tilde{F}\right|_{\mathscr{A}}(x)+\left.g^{2} \operatorname{tr}(G \tilde{G})\right|_{A}(x)\right]
\end{aligned}
$$

where

$$
\tilde{F}_{\mu \nu}=\frac{1}{2} \varepsilon_{\mu v \rho \sigma} F_{\rho \sigma} \quad \tilde{G}_{\mu \nu}^{a}=\frac{1}{2} \varepsilon_{\mu v \rho \sigma} G_{\rho \sigma}^{a} .
$$

Eq. (2.16) shows that the lattice action (2.4) leads to a continuum effective theory with vacuumangle $\hat{\theta}_{u}+\hat{\theta}_{d}$. In general, $\hat{\theta}_{u}+\hat{\theta}_{d}$ is not simply $\propto \theta_{u}+\theta_{d}$ due to e.m. interactions implying $\frac{Z_{s}^{u}}{Z_{P}^{u}} \neq \frac{Z_{s}^{d}}{Z_{P}^{d}}$. However from eq. (2.14) one checks that at maximal twist (e.g. $\theta_{u}=-\theta_{d}=\frac{\pi}{2}$ ) the continuum effective theory has $\hat{\theta}_{u}=-\hat{\theta}_{d}=\frac{\pi}{2}$, hence vanishing vacuum-angle and no undesired parity violation. In other words once $M_{c r}$ is determined we get $\mathrm{Q}(\mathrm{C}+\mathrm{E}) \mathrm{D}$ for two non-degenerate quarks.

\section{Fixing the critical mass in the twisted lattice $Q(C+E) D$ theory}

In our setting with two non-degenerate flavours we first have to non-perturbatively determine the critical mass, $M_{c r}$ (see eq.2.3), appearing in eq. (2.1). As we shall work at first order in $\alpha_{e m}$, we need to accordingly expand the parameters $m_{c r}$ and $\tilde{m}_{c r}$ of eq.(2.3) in powers of $\alpha_{e m}$, obtaining

$$
m_{c r}=m_{c r}^{L Q C D}+\alpha_{e m} \frac{\delta_{e m}\left(g^{2}\right)}{a}+\mathrm{O}\left(\alpha_{e m}^{2}\right) \quad \tilde{m}_{c r}=\alpha_{e m} \frac{\tilde{\delta}_{e m}\left(g^{2}\right)}{a}+\mathrm{O}\left(\alpha_{e m}^{2}\right),
$$

where $m_{c r}^{L Q C D}=w\left(g^{2}\right) / a$ is the critical mass of the isosymmetric theory. From now on a suitable e.m. gauge fixing and procedure (e.g. $\mathrm{QED}_{L}$ ) for removal of the photon zero mode are assumed. Following the strategy outlined in $[13,8,10]$ one can determine $M_{c r}$ by enforcing the chiral WTIs 
of $\mathrm{Q}(\mathrm{C}+\mathrm{E}) \mathrm{D}$. For instance, with obvious notations for quark bilinears in $\psi$-basis (e.g. $P^{1,2,3}=$ $\left.\bar{\psi} \gamma_{5} \frac{\tau^{1,2,3}}{2} \psi\right)$ and $\delta q \equiv q_{u}-q_{d}=1$ a way to fix $M_{c r}$ might be to enforce the formal continuum relations

$$
\begin{aligned}
& \left\langle\partial_{\mu} V_{\mu}^{1}(x) P^{2}(0)\right\rangle+e \delta q\left\langle\mathscr{A}_{\mu} V_{\mu}^{2}(x) P^{2}(0)\right\rangle-2 i \varepsilon\left\langle S^{2}(x) P^{2}(0)\right\rangle=0 \\
& \left\langle\partial_{\mu} A_{\mu}^{1}(x) S^{1}(0)\right\rangle-e \delta q\left\langle\mathscr{A}_{\mu} A_{\mu}^{2}(x) S^{1}(0)\right\rangle-2 \mu\left\langle P^{1}(x) S^{1}(0)\right\rangle=0 .
\end{aligned}
$$

This procedure is rather practical as the flavour structure of the two relations is such that, once $m_{c r}^{L Q C D}$ is known, it is possible to separately determine $\delta_{e m}$ (from eq. (3.2)) and $\tilde{\delta}_{e m}$ (from eq. (3.3)) ${ }^{2}$.

A look at eqs. (3.2)) and (3.3) shows that only parity violating correlators come into play. This fact together with a reconsideration of the condition usually employed in LQCD to determine the critical mass suggests a numerically simpler way to fix $m_{c r}$ and $\tilde{m}_{c r}$.

To explain the idea we first discuss the situation one meets in twisted mass LQCD. In this case the LQCD Symanzik effective action [14] for $m_{0}$ out of its critical value takes the form

$$
\Gamma_{L Q C D}=\int d^{4} x\left\{L_{4}^{Q C D}(x)+\left(w\left(g^{2}\right) / a-m_{0}\right)\left[\bar{\psi} i \gamma_{5} \tau_{3} \psi\right](x)+a L_{5}(x)+\ldots\right\} .
$$

The undesired term $\propto\left[\bar{\psi} i \gamma_{5} \tau_{3} \psi\right]$ in (3.4) can be eliminated from $\Gamma_{L Q C D}$ by enforcing the condition $\sum_{\vec{x}}\left\langle V_{0}^{1}(x) P^{2}(0)\right\rangle_{M_{0}}^{l a t t}=0$ (for $\left.x_{0} \gg a\right)^{3}$. Indeed from the Symanzik expansion we obtain

$$
\begin{aligned}
& \left\langle V_{0}^{1}(x) P^{2}(0)\right\rangle_{m_{0}}^{l a t t}=\left\langle V_{0}^{1}(x) P^{2}(0)\right\rangle^{L_{4}^{Q C D}}+\left(w\left(g^{2}\right) / a-m_{0}\right) \int d^{4} y\left\langle V_{0}^{1}(x) P^{2}(0)\left[\bar{\psi} i \gamma_{5} \tau_{3} \psi\right](y)\right\rangle^{L_{4}^{Q C D}}+\mathrm{O}(a)= \\
& =\left(w\left(g^{2}\right) / a-m_{0}\right) \int d^{4} y\left\langle V_{0}^{1}(x) P^{2}(0)\left[\bar{\psi} i \gamma_{5} \tau_{3} \psi\right](y)\right\rangle^{Q C C D}+\mathrm{O}(a),
\end{aligned}
$$

where last equality follows from $\left\langle V_{0}^{1}(x) P^{2}(0)\right\rangle^{L_{4}^{Q C D}}=0$ in the parity-and-flavour invariant $L_{4}^{Q C D}$.

To determine $M_{c r}$ in $\mathrm{LQ}(\mathrm{C}+\mathrm{E}) \mathrm{D}$ we can use a similar method. Working for simplicity with $\varepsilon=0$ in (2.1) the Symanzik low energy action of LQ(C+E)D at $M_{0} \equiv m_{0} 11+\tilde{m}_{0} \tau^{3} \neq M_{c r}$ reads

$$
\Gamma_{L Q(C+E) D}=\int d^{4} x\left\{L_{4}^{Q(C+E) D}(x)+\left(m_{c r}-m_{0}\right)\left[\bar{\psi} i \gamma_{5} \tau_{3} \psi\right](y)+\left(\tilde{m}_{c r}-\tilde{m}_{0}\right)\left[\bar{\psi} i \gamma_{5} \psi\right](x)+a L_{5}(x)+\ldots\right\},
$$

where we are simultaneously expanding in $m_{0}-m_{c r}, \tilde{m}_{0}-\tilde{m}_{c r}$ and $a$ around the continuum theory $L_{4}^{Q(C+E) D}$ with $\hat{m}_{u}=\hat{m}_{d}=0$ (but $\hat{\mu}_{u}>0, \hat{\mu}_{d}<0$ and $\alpha_{e m} \neq 0$ ). Our claim is that $m_{c r}$ and $\tilde{m}_{c r}$ can be determined to first order in $\alpha_{e m}$ by enforcing the conditions

$$
\sum_{\vec{x}}\left\langle V_{0}^{1}(x) P^{2}(0)\right\rangle_{M_{0}}^{\text {latt }}=0, \quad \sum_{\vec{x}}\left\langle S^{1}(x) P^{1}(0)\right\rangle_{M_{0}}^{\text {latt }}=0, \quad x_{0} \gg a .
$$

In fact, based on the effective action (3.6) and using parity invariance of $L_{4}^{Q(C+E) D}$, we can write

$$
\begin{aligned}
& \left\langle V_{0}^{1}(x) P^{2}(0)\right\rangle_{M_{0}}^{l a t t}=\left.\left(m_{c r}^{L Q C D}+\alpha_{e m} \delta_{e m} a^{-1}-m_{0}\right) \int d^{4} z\left\langle V_{0}^{1}(x) P^{2}(0) \bar{\psi} i \gamma_{5} \tau_{3} \psi(z)\right\rangle\right|^{L_{4}^{Q(C+E) D}}+ \\
& \quad+\left.\left(\alpha_{e m} \tilde{\delta}_{e m} a^{-1}-\tilde{m}_{0}\right) \int d^{4} z\left\langle V_{0}^{1}(x) P^{2}(0) \bar{\psi} i \gamma_{5} \psi(z)\right\rangle\right|^{Q(C+E) D}+\mathrm{O}(a) \\
& \left\langle S^{1}(x) P^{1}(0)\right\rangle_{M_{0}}^{l a t t}=\left.\left(\alpha_{e m} \tilde{\delta}_{e m} a^{-1}-\tilde{m}_{0}\right) \int d^{4} z\left\langle S^{1}(x) P^{1}(0) \bar{\psi} i \gamma_{5} \psi(z)\right\rangle\right|_{4} ^{Q(C+E) D}+ \\
& \quad+\left.\left(m_{c r}^{L Q C D}+\alpha_{e m} \delta_{e m} a^{-1}-m_{0}\right) \int d^{4} z\left\langle S^{1}(x) P^{1}(0) \bar{\psi} i \gamma_{5} \tau_{3} \psi(z)\right\rangle\right|^{Q C+E) D}+\mathrm{O}(a) .
\end{aligned}
$$

\footnotetext{
${ }^{2}$ Another way to fix $M_{c r}$ is to impose one chiral WTI (as was done in the electroquenched approximation in [2]) and then take as second condition the one arising from the minimization of $m_{\pi}^{ \pm}$with respect to $m_{u, d}$ (as is detailed in [11]).

${ }^{3}$ This condition in Wilson twisted mass LQCD returns what in ref. [15] was called the "optimal" critical mass.
} 
Assuming that $m_{c r}^{L Q C D}$ is already known, for our purposes $\delta m_{0} \equiv m_{0}-m_{c r}^{L Q C D}$ and $\tilde{m}_{0}$ can be viewed as $\mathrm{O}\left(\alpha_{e m}\right)$ quantities. Expanding the r.h.s. of eqs. (3.8)-(3.9) in $\alpha_{e m}$ to leading order we find

$$
\begin{aligned}
& \left\langle V_{0}^{1}(x) P^{2}(0)\right\rangle_{M_{0}}^{l a t t}=\left.\left(\alpha_{e m} \delta_{e m} a^{-1}-\delta m_{0}\right) \int d^{4} z\left\langle V_{0}^{1}(x) P^{2}(0) \bar{\psi} i \gamma_{5} \tau_{3} \psi(z)\right\rangle\right|^{L_{4}^{Q C D}}+\mathrm{O}(a) \\
& \left\langle S^{1}(x) P^{1}(0)\right\rangle_{M_{0}}^{l a t t}=\left.\left(\alpha_{e m} \tilde{\delta}_{e m} a^{-1}-\tilde{m}_{0}\right) \int d^{4} z\left\langle S^{1}(x) P^{1}(0) \bar{\psi} i \gamma_{5} \psi(z)\right\rangle\right|^{Q L_{4}^{Q C D}}+\mathrm{O}(a),
\end{aligned}
$$

with all $\mathrm{O}\left(\alpha_{e m}^{2}\right)$ contributions in the r.h.s neglected. Hence the contributions from the correlators in the r.h.s. of eqs. (3.8) and (3.9) are evaluated in the continuum $L_{4}^{Q C D}$ theory (those in the first line, which are $\mathrm{O}\left(\alpha_{e m}\right)$ ) or ignored (those in the second line, which are $\left.\mathrm{O}\left(\alpha_{e m}^{2}\right)\right)$. On the other hand the lattice correlators in the 1.h.s. of eqs. (3.10)-(3.11) must be computed to $\mathrm{O}\left(\alpha_{e m}\right)$ in $\mathrm{LQ}(\mathrm{C}+\mathrm{E}) \mathrm{D}$. We thus see that enforcing the conditions (3.7) allows to set $a \delta m_{0}$ and $a \tilde{m}_{0}$ equal to $\alpha_{e m} \delta_{e m}$ and $\alpha_{e m} \tilde{\delta}_{e m}$, respectively, thereby determining $M_{c r}$ (eqs. (2.3) and (3.1)) at the desired order.

\section{LIB effects in $\mathbf{Q}(\mathbf{C}+\mathbf{E}) \mathbf{D}$ with unquenched $u, d$, s and $c$ : a simulation strategy}

Due to e.m. gauge invariance the lattice action for maximally twisted quark pairs $\psi^{\ell}=(u, d)$ and $\psi^{h}=(c, s)$ should in principle involve only identity and $\tau^{3}$ Pauli matrices in flavour space, i.e.

$S_{33}^{\ell h}=a^{4} \sum_{x}\left\{\bar{\psi}^{\ell}(x)\left[\gamma \cdot \widetilde{\nabla}-i \gamma_{5} \tau_{3} W_{\mathrm{cr}}+\mu_{\ell}+\tau_{3} \varepsilon_{\ell}\right] \psi^{\ell}(x)+\bar{\psi}^{h}(x)\left[\gamma \cdot \widetilde{\nabla}-i \gamma_{5} \tau_{3} W_{\mathrm{cr}}+\mu_{h}+\tau_{3} \varepsilon_{h}\right] \psi^{h}(x)\right\}$.

As remarked above, the occurrence of a $i \gamma_{5} \tau^{3}$ structure in the twisted critical Wilson term leads to a complex fermionic determinant, making problematic direct Monte Carlo simulations of this lattice formulation of $\mathrm{Q}(\mathrm{C}+\mathrm{E}) \mathrm{D}^{4}$. The use of the RM123 method for LIB corrections to physical observables substantially alleviates this problem because the relevant info is extracted from renormalized correlation functions with insertions of suitable operators (e.m. currents, isospin breaking quark densities) to be evaluated in the isosymmetric theory [2], v.i.z.

$\left.\langle\hat{\mathscr{O}}\rangle\right|_{\hat{g}_{i}} ^{\text {latt }}=\left.\langle\hat{\mathscr{O}}\rangle\right|_{\hat{g}_{i}^{0}} ^{\text {latt }}+\left.\hat{e}^{2} \frac{\partial\langle\hat{\mathscr{O}}\rangle}{\partial \hat{e}^{2}}\right|_{\hat{g}_{i}^{0}} ^{l a t t}+\left.\left[\hat{g}_{s}^{2}-\left(\frac{Z_{g_{s}}}{Z_{g_{s}}^{0}} \hat{g}_{s}^{0}\right)^{2}\right] \frac{\partial\langle\hat{\mathscr{O}}\rangle}{\partial \hat{g}_{s}^{2}}\right|_{\hat{g}_{i}^{0}} ^{\text {latt }}+\left.\left[\hat{\mu}_{f}-\left(\frac{Z_{\mu_{f}}}{Z_{\mu_{f}}^{0}} \hat{\mu}_{f}^{0}\right)\right] \frac{\partial\langle\hat{\mathscr{O}}\rangle}{\partial \hat{\mu}_{f}}\right|_{\hat{g}_{i}^{0}} ^{\text {latt }}$,

where $\hat{g}_{i}^{0}\left(\hat{g}_{i}\right)$ denote the renormalized couplings of the isosymmetric (full) theory $\left(g_{i}=\left\{e, g_{s}, \mu_{f}\right\}\right.$, with $\mu_{f}$ being the $f$-quark mass (at maximal twist), and $\hat{\mathscr{O}}$ are renormalized multilocal operators. The derivatives with respect to renormalized couplings give rise to space-time integrated insertions of the corresponding renormalized operators in the correlation functions of the isosymmetric theory. The maximally twisted mass regularization of the latter admits indeed a real positive fermionic determinant in the $(u, d)$-sector (where now $\varepsilon_{\ell}=0$ ) and, provided a suitable mixed action approach (see below) is employed, also in $(c, s)$-sector, where the large mass splitting term $\propto \varepsilon_{h}$ occurs.

The evaluation of the renormalized correlation functions of interest should be in principle carried out in the theory with action $S_{33}^{\ell h}$ at $e=\varepsilon_{\ell}=0$. But having still $\varepsilon_{h} \neq 0$ the simulation is made problematic by the complex (c-s sector) fermionic determinant. However, up to negligible

\footnotetext{
${ }^{4}$ In the absence of e.m. interactions one can use a lattice action (which we might call $S_{13}^{\ell h}$ ) having instead a $i \gamma_{5} \tau^{1}$ matrix in the twisted critical Wilson term: in this case the Dirac operator is $\gamma_{5} \tau^{3}$-Hermitean and has a real determinant [10].
} 
$\mathrm{O}\left(a^{2}\right)$ lattice artifacts, precisely the same set of renormalized correlation functions can also be evaluated by using the isosymmetric lattice action (mixed action approach)

$$
\begin{aligned}
S^{m i x}= & \left.S_{Y M}(U, E)\right|_{e=0}+\left.S_{33}^{\ell h}\left(\mu_{\ell}, 0, \mu_{h}, \varepsilon_{h}\right)\right|_{e=0}+\left.a^{4} \sum_{x} \Phi_{g h}^{\dagger}\left[\gamma \cdot \widetilde{\nabla}-i \gamma_{5} \tau_{3} W_{\mathrm{cr}}+\mu_{h}+\tau_{3} \varepsilon_{h}\right] \Phi_{g h}\right|_{e=0}+ \\
& +\left.a^{4} \sum_{x} \bar{\psi}_{\text {sea }}^{h}(x)\left[\gamma \cdot \widetilde{\nabla}-i \gamma_{5} \tau_{1} W_{\mathrm{cr}}+\mu_{h}+\tau_{3} \varepsilon_{h}^{\text {sea }}\right] \psi_{\text {sea }}^{h}(x)\right|_{e=0}
\end{aligned}
$$

where $S_{Y M}(U, E)$ denotes the gluon and photon lattice action and $S_{33}^{\ell h}$ is given in eq. (4.1). The virtual "sea" effects of the quark fields $\psi_{h}, \bar{\psi}_{h}$ are canceled by those of the complex ghost field $\Phi_{h}$ but reintroduced through the fields $\psi_{h}^{\text {sea }}, \bar{\psi}_{h}^{\text {sea }}$. Of course the mixed action theory $S^{\text {mix }}$ must be simulated at (fixed as $a \rightarrow 0$ ) renormalized parameters $\left(\hat{g}_{i}^{0}\right)$ matching those of the theory $\left[S_{Y M}(U, E)+\right.$ $\left.S_{33}^{\ell h}\left(\mu_{\ell}, 0, \mu_{h}, \varepsilon_{h}\right)\right]\left.\right|_{e=0}$ one should have studied in principle, and in particular with equal values for the valence and sea renormalized masses of each quark flavour. In the isosymmetric theory this implies working with equal values of the valence and sea bare mass parameters, except for $\varepsilon_{h}=Z_{P^{0}} Z_{S}^{-1} \varepsilon_{h}^{\text {sea }}$. Only e.m. corrections will give rise to the more complicated pattern of eq. (2.13).

The proof of the statements above is straightforward [12] and can be given along the lines of ref. [9]. We note that the form of the valence $c-s$ sector in the action (4.3) is such that within the RM123 approach for LIB corrections to hadronic observables one has to evaluate precisely the same set of Wick contractions that should be computed if the action $S_{33}^{\ell h}$ were adopted, but employing gauge ensembles that are generated with no "complex phase problems". This is so because $\varepsilon_{\ell}=0$ and the $c$-s sea quark effects stem from the action term in second line of eq. (4.3).

Acknowledgements We thank V. Lubicz, G. Martinelli and S. Simula for stimulating discussions. R.F. and N.T. acknowledge support through the grant "Uncovering Excellence 2014 - LIBETOV".

\section{References}

[1] G. M. de Divitiis et al., JHEP 1204 (2012) 124.

[2] G. M. de Divitiis et al. [RM123 Collaboration], Phys. Rev. D 87 (2013) 114505.

[3] B. Blossier et al. [ETM Collaboration], Phys. Rev. D 82 (2010) 114513.

[4] R. Baron et al. [ETM Collaboration], JHEP 1008 (2010) 097.

[5] N. Tantalo et al., https://conference.ippp.dur.ac.uk/event/470/session/15/contribution/313.

[6] V. Lubicz et al., arXiv:1610.09668 [hep-lat].

[7] N. Carrasco et al., Phys. Rev. D 91 (2015) 074506.

[8] R. Frezzotti and G. C. Rossi, JHEP 0408 (2004) 007.

[9] R. Frezzotti and G. C. Rossi, JHEP 0410 (2004) 070.

[10] R. Frezzotti and G. C. Rossi, Nucl. Phys. Proc. Suppl. 128 (2004) 193.

[11] D. P. Horkel and S. R. Sharpe, Phys. Rev. D 92 (2015) 074501 and Phys. Rev. D 92 (2015) 094514.

[12] R. Frezzotti et al., in preparation.

[13] M. Bochicchio, L. Maiani, G. Martinelli, G. C. Rossi and M. Testa, Nucl. Phys. B 262 (1985) 331.

[14] K. Symanzik, Nucl. Phys. B 226 (1983) 187 and 205.

[15] R. Frezzotti, G. Martinelli, M. Papinutto and G. C. Rossi, JHEP 0604 (2006) 038. 